\title{
INVERSE SYSTEMS OF ABSOLUTE RETRACTS AND ALMOST CONTINUITY
}

\author{
VLADIMIR N. AKIS
}

\begin{abstract}
Suppose that $Y$ is the inverse limit of a sequence of absolute retracts such that each bonding map is a retraction. We show that $Y$ is the almost continuous retract of the Hilbert cube. It follows that $Y$, the cone over $Y$, the suspension of $Y$, and the product of $Y$ with any absolute retract must have the fixed point property.
\end{abstract}

Introduction. Throughout this paper $X$ and $Y$ will denote topological spaces. A map is a continuous function. When $f: X \rightarrow Y$ may not be continuous, we refer to it simply as the function $f$. A space $X$ has the fixed point property if for each map $f: X \rightarrow X$ there exist $x \in X$ such that $f(x)=x$. A continuum is a compact connected metric topological space. An absolute retract (AR) is a retract of the Hilbert cube.

The fixed point property has been the subject of intense investigation. Many surprising results have been revealed, but many questions still remain unanswered.

J. Stallings [7] defined a class of functions, which he named almost continuous, for the purpose of studying the fixed point property.

The graph of a function $f: X \rightarrow Y$ is the subset of $X \times Y$ consisting of the points $(x, f(x))$; this set will be symbolized $\Gamma(f)$.

DEFINITION 1 [7, p. 252]. A function $f: X \rightarrow Y$ is almost continuous if for each open subset $U$ of $X \times Y$ such that $\Gamma(f) \subset U$, there exists a map $g: X \rightarrow Y$ such that $\Gamma(g) \subset U$.

DEFINITION 2. If $Y \subset X$ and $r: X \rightarrow Y$ is an almost continuous function such that $r(X)=Y$ and for all $x \in Y, r(x)=x$, then $r$ is called a quasi retraction and $Y$ is called a quasi retract of $X$.

DEFinition 3. If $Y \subset X$ and $r: X \rightarrow Y$ is an almost continuous function such that $r(X)=Y$ and $r(x)=x$ for all $x \in Y$, then $r$ is called an almost continuous retraction and $Y$ is called an almost continuous retract of $X .^{1}$

DEFINITION 4 [1, P. 48]. A compact metric space $Y$ is an absolute quasi retract (AQR) if $Y$ is homeomorphic to a quasi retract of the Hilbert cube.

THEOREM $1[\mathbf{1}]$. Every $A Q R$ has the fixed point property.

THEOREM $2[\mathbf{1}$ ]. If $X$ is an $A R$ and $Y$ an $A Q R$, then $X \times Y$ is an $A Q R$.

Received by the editors September 24, 1984 and, in revised form, January 2, 1985. Presented to the Topology Spring Conference, 1984, at Auburn University, Auburn, Alabama.

1980 Mathematics Subject Classification. Primary 54C10, 54B25; Secondary 54C55, 54H15, 54H25.

${ }^{1}$ In the literature both almost continuous retractions and quasi retractions have been called almost continuous retractions. We make the distinction because even though almost continuous retractions are quasi retractions, the converse is not true. We refer the interested reader to [3] and $[\mathbf{1}]$. 
COROLlary. If $X$ is an $A R$ and $Y$ an $A Q R$, then $X \times Y$ has the fixed point property.

Let $I=\{t: 0 \leq t \leq 1\}$. For any space $X$ the cone $T X$ over $X$ is the quotient space $(X \times I) / R$, where $R$ is the equivalence relation $(x, t) \sim(y, s)$ if and only if $t=s=1$, or $x=y$ and $t=s$, for all $x, y \in X$ and $s, t \in I$. The suspension $S X$ of $X$ is the quotient space $(X \times I) / Q$, where $Q$ is the equivalence relation $(x, t) \sim(y, s)$ if and only if $t=s=1$ or $t=s=0$ or $x=y$ and $t=s$, for $x, y \in X$ and $s, t \in I$.

THEOREM $3[\mathbf{1}]$. If $X$ is an $A Q R$, then $T X$ and $S X$ are $A Q R s$.

COROLlary. If $X$ is an $A Q R$, then $T X$ and $S X$ have the fixed point property.

Given a sequence of topological spaces $Y_{1}, Y_{2}, \ldots$ and maps $g[j, i]: Y_{j} \rightarrow Y_{i}$ where $i<j$, the set $\left\{Y_{i} ; g[j, k]\right\}$ is called an inverse system. The maps $g[j, k]$ are called the bonding maps of the inverse system. Let $Y$ be the set of all points $\left\langle y_{1}, y_{2}, \ldots\right\rangle$ in $\prod Y_{i}$ such that, for $i<j, y_{i}=g[j, i]\left(y_{j}\right)$. The set $Y$, considered as a subspace of $\prod Y_{i}$, is called the inverse limit of $\left\{Y_{i} ; g[j, k]\right\}$.

We prove that if $Y$ is the inverse limit of $\left\{Y_{i} ; r[j, i]\right\}$ where each $Y_{i}$ is an absolute retract and each bonding map $r[j, i]$ is a retraction, then $Y$ is homeomorphic to an almost continuous retract of the Hilbert cube. Therefore $Y$ is an AQR.

The main results. An almost continuous function $f: X \rightarrow Y$ is a function approximated by maps in the sense of Definition 1 . If however, $X$ and $Y$ are compact metric spaces there is a characterization of almost continuity in terms of sequences of maps.

DEFINITION 5. A sequence $\left\{f_{n}\right\}$ of function of $X$ into $Y$ almost continuously approximates a function $f: X \rightarrow Y$ if for every sequence $\left\{x_{n}\right\} \subset X$, such that $f_{n}\left(x_{n}\right) \neq f\left(x_{n}\right)$, there exists a subsequence $\left\{x_{n_{i}}\right\} \subset\left\{x_{n}\right\}$ and $x \in X$ such that $x_{n_{i}} \rightarrow x$ and $f_{n_{i}}\left(x_{n_{i}}\right) \rightarrow f(x)$.

THEOREM $4[\mathbf{1}]$. Assume $X$ and $Y$ are compact metric spaces. Then $f: X \rightarrow Y$ is almost continuous if and only if there exists a sequence of maps $f_{n}: X \rightarrow Y$ such that $\left\{f_{n}\right\}$ almost continuously approximates $f$.

Henceforth we will use Theorem 4 for proving that a given function is almost continuous.

THEOREM 5. Let $(X, \rho)$ be a compact metric space. Suppose $X=X_{1} \supset X_{2} \supset$ .. つ $X_{n} \supset X_{n+1} \supset \cdots$ is a decreasing sequence of compact subsets of $X$ such that $X_{n+1} \subset \operatorname{int} X_{n}$. Also suppose that $M_{1} \subset M_{2} \subset \cdots \subset M_{n} \subset M_{n+1} \subset \cdots$ is an increasing sequence of absolute retracts such that $M_{n} \subset X_{n}$. If there exist maps $r_{n}: X_{n} \rightarrow M_{n}$ such that $\rho\left(x, r_{n}(x)\right)<1 / n$, then $\bigcap X_{i}$ is an almost continuous retract of $X$.

Proof. Let $f_{1}=r_{1}$. Thus $f_{1}$ is a map of $X_{1}$ into $M_{1}$. Inductively we define maps $f_{n}: X_{1} \rightarrow M_{n}$ as follows. Given $f_{k}: X_{1} \rightarrow M_{k}$ let $G_{k}$ be open in $X_{1}$ such that $X_{k+1} \subset G_{k} \subset X_{k}$ and let $F_{k}=\left(X_{1} \sim G_{k}\right) \cup X_{k+1}$. Next define a map $g_{k}: F_{k} \rightarrow M_{k+1}$ as follows:

$$
g_{k}(x)= \begin{cases}f_{k}(x) & \text { if } x \in X_{1} \sim G_{k}, \\ r_{k+1}(x) & \text { if } x \in X_{k+1}\end{cases}
$$

Now extend $g_{k}$ to a map $f_{k+1}: X_{1} \rightarrow M_{k+1}$. 
Consider the function $r: X_{1} \rightarrow \bigcap X_{i}$ defined as

$$
r(x)= \begin{cases}x & \text { if } x \in \bigcap_{i} \\ f_{n+1}(x) & \text { if } x \in X_{n} \sim X_{n+1}\end{cases}
$$

We prove that $r: X_{1} \rightarrow \bigcap X_{i}$ is almost continuous by showing that $\left\{f_{n}\right\}$ almost continuously approximates $r$.

Choose a sequence $\left\{x_{n}\right\}$ in $X_{1}$, such that $f_{n}\left(x_{n}\right) \neq r\left(x_{n}\right)$. Since $r(x)=f_{n}(x)$ for every $x \in X \sim X_{n}$, we conclude that $x_{n} \in X_{n}$. Therefore $f_{n}\left(x_{n}\right)=r_{n}\left(x_{n}\right)$.

Let $\left\{x_{n_{i}}\right\}$ be a convergent subsequence of $\left\{x_{n}\right\}$, with $x_{n_{i}} \rightarrow x \in \bigcap X_{i}$. Since $\rho\left(r_{n}\left(x_{n}\right), x_{n}\right)<1 / n$, we conclude that $r_{n_{i}}\left(x_{n_{i}}\right) \rightarrow x$, hence $f_{n_{i}}\left(x_{n_{i}}\right) \rightarrow x$. Also $r(x)=x$ because $x \in \bigcap X_{i}$, it follows that $f_{n_{i}}\left(x_{n_{i}}\right) \rightarrow r(x)$. Therefore $\left\{f_{n}\right\}$ almost continuously approximates $r$. Thus $r: X \rightarrow \bigcap X_{i}$ is an almost continuous retraction. And the proof of Theorem 5 is complete.

Next we consider inverse systems of absolute retracts with bonding maps which are retractions.

THEOREM 6. Let $\left\{Y_{i} ; r[j, k]\right\}$ be an inverse system such that each $Y_{i}$ is an absolute retract, and if $i<j$ then $Y \subset Y_{j}$, and $r[j, k]: Y_{j} \rightarrow Y_{i}$ is a retraction. Then the inverse limit of $\left\{Y_{i} ; r[j, i]\right\}$ is an almost continuous retract of $\prod Y_{i}$.

ProOF. Let $Y$ denote the inverse limit of $\left\{Y_{i} ; r[j, i]\right\}$. Let $X_{1}=\prod Y_{i}$. For $n=2,3,4, \ldots$ let $X_{n}$ be the set of all points $\left\langle x_{i}\right\rangle=\left\langle x_{1}, x_{2}, \ldots\right\rangle$ in $\prod Y_{i}$ such that if $i<j \leq n$ then $\rho_{i}\left(x_{i}, r[j, i]\left(x_{j}\right)\right) \leq 2^{-n}$, where $\rho_{i}$ denotes the metric of $Y_{i}$.

By a routine argument, each $X_{n}$ is compact, and each $X_{n+1} \subset \operatorname{int} X_{n}$; and, clearly, $Y=\bigcap X_{n}$.

For $n=1,2,3, \ldots$ let $M_{n}$ be the set of all points $\left\langle x_{i}\right\rangle$ in $\prod Y_{i}$ such that if $i<n$ then $x_{i}=r[n, i]\left(x_{n}\right)$ and if $i \geq n$ then $x_{i}=x_{n}$. Then $M_{n} \subset M_{n+1}$, and each $M_{n}$ is an AR since it is homeomorphic to $Y_{n}$.

Next we define the maps $r_{n}: X_{n} \rightarrow M_{n}$ as follows:

$$
r_{n}\left\langle x_{i}\right\rangle= \begin{cases}r[n, i]\left(x_{n}\right) & \text { if } i<n, \\ x_{n} & \text { if } i \geq n .\end{cases}
$$

Let $\rho$ denote the metric of $\prod Y_{i}$, defined by $\rho\left(\left\langle x_{i}\right\rangle,\left\langle y_{i}\right\rangle\right)=\sum 2^{-i} \rho_{i}\left(x_{i}, y_{i}\right)$. Then for $\left\langle x_{i}\right\rangle \in X_{n}$,

$$
\rho\left(\left\langle x_{i}\right\rangle, r_{n}\left\langle x_{i}\right\rangle\right)=\sum_{i=1}^{n-1} \frac{\rho_{i}\left(x_{i}, r[n, i]\left(x_{n}\right)\right)}{2^{i}}+\sum_{i=n}^{\infty} \frac{\rho_{i}\left(x_{i}, x_{n}\right)}{2^{i}} .
$$

We may assume with no loss of generality that $\rho_{i}(x, y) \leq \frac{1}{2}$ for each $x, y \in Y_{i}$ and $i=1,2,3, \ldots$. Hence

$$
\rho\left(\left\langle x_{i}\right\rangle, r_{n}\left\langle x_{i}\right\rangle\right) \leq \frac{1}{2^{n}} \sum_{i=1}^{n-1} \frac{1}{2^{i}}+\frac{1}{2^{n}}<\frac{1}{2^{n-1}}<\frac{1}{n} .
$$

It follows from Theorem 5 that $Y$ is an almost continuous retract of $\prod Y_{i}$.

COROLLARY. Let $Y$ be the inverse limit of $\left\{Y_{i} ; r[j, i]\right\}$ as described in Theorem 6. Then $Y$ is homeomorphic to an almost continuous retract of the Hilbert cube $H$.

PROOF. Since each $Y_{i}$ is an AR, then $\prod Y_{i}$ is an AR [2]. Let $r: H \rightarrow \prod Y_{i}$ be a retraction and let $q: \prod Y_{i} \rightarrow Y$ be an almost continuous retraction. Then by Proposition 4 of [7, p. 261], $q r: H \rightarrow Y$ is an almost continuous retraction. 
COROLlaRY. Let $Y$ be the inverse limit of $\left\{Y_{i} ; r[j, i]\right\}$ as described in Theorem 6. Then $Y$, the cone over $Y$, the suspension of $Y$, and the product of $Y$ with an $A R$ must have the fixed point property.

PROOF. This follows from the preceding corollary and the corollaries of Theorems 2 and 3 .

Concluding remarks and questions. A map $f: X \rightarrow Y$ is universal if for any continuous map : $X \rightarrow Y$ there exists $x \in X$ such that $g(x)=f(x)$. W. Holsztynski [4] has shown that if $Y$ is the inverse limit of $\left\{Y_{i} ; g[j, i]\right\}$, where each $Y_{i}$ is an AR and each bonding map $g[j, i]$ is universal, then $Y$ has the fixed point property. It is easy to see that if $Y$ has the fixed point property, and if $r: X \rightarrow Y$ is a retraction, then $r$ is universal. Thus our last corollary also follows from Holsztynski's theorem.

In view of Holsztynski's result one might hope to get a stronger version of Theorem 6 , by allowing the bonding maps $r[j, i]$ to be universal. However, this cannot be done. K. Kellum [5] proved that: Given a 2nd countable space $Y$, there exists a Peano continuum $P$ such that $Y$ is the image of a surjective almost continuous function $f: P \rightarrow Y$ if and only if $Y$ is almost Peano. That $Y$ is almost Peano means that for each finite collection of nonempty open subsets of $Y$, there is a Peano continuum in $Y$ which intersects each of them. It follows that if $Y$ is the almost continuous image of the Hilbert cube then $Y$ is almost Peano. However, the pseudoarc [6] is not almost Peano, since it contains no Peano subcontinuum. But the pseudoarc is the inverse limit of arcs. And since every map of an arc onto itself is universal [4], the pseudoarc is the inverse limit of absolute retracts with universal bonding maps. We conclude that there exists an example of a space, which is the inverse limit of absolute retracts with universal bonding maps, that is not an almost continuous retract of the Hilbert cube.

However, it is not known if the pseudoarc is an AQR. Consequently, it is natural to ask the following

Question. If $Y$ is the inverse limit of $\left\{Y_{i} ; g[j, i]\right\}$, where each $Y_{i}$ is an absolute retract and each $g[j, i]$ is universal, is $Y$ an AQR?

The author gratefully acknowledges conversations about topics in this paper with Professors C. L. Hagopian and M. M. Marsh.

\section{REFERENCES}

1. V. N. Akis, Fixed point theorems and almost continuity, Fund. Math. 121 (1984), 134-142.

2. K. Borsuk, Theory of retracts, Monograf. Mat., Tom 44, PWN, Warsaw, 1967.

3. B. D. Garrett, Almost continuous retracts, General Topology and Modern Analysis, Academic Press, New York, 1981, pp. 229-238.

4. W. Holsztynski, Universal mappings and fixed point theorems, Bull. Acad. Polon. Sci. Sér. Sci. Math. 15 (1967), 433-438.

5. K. R. Kellum, Almost continuous images of Peano continua, Topology Appl. 11 (1980), 293-296.

6. E. E. Moise, An indecomposable plane continuum which is homeomorphic to each of its nondegenerate subcontinua, Trans. Amer. Math. Soc. 63 (1948), 581-594.

7. J. Stallings, Fixed point theorems for connectivity maps, Fund. Math. 47 (1959), 249-263.

Department of Mathematics and Computer SCience, California State UniVERSITY, LOS ANGELES, CALIFORNIA 90032 